\title{
Efficacy of mindfulness-based cognitive therapy in relation to prior history of depression: randomised controlled trial ${ }^{\dagger}$
}

\author{
Nicole Geschwind, Frenk Peeters, Marcus Huibers, Jim van Os and Marieke Wichers
}

\section{Background}

There appears to be consensus that patients with only one or two prior depressive episodes do not benefit from treatment with mindfulness-based cognitive therapy (MBCT).

\begin{abstract}
Aims
To investigate whether the effect of MBCT on residual depressive symptoms is contingent on the number of previous depressive episodes (trial number NTR1084).
\end{abstract}

\section{Method}

Currently non-depressed adults with residual depressive symptoms and a history of depression $(\leqslant 2$ prior episodes: $n=71 ; \geqslant 3$ episodes: $n=59$ ) were randomised to MBCT $(n=64)$ or a waiting list (control: $n=66)$ in an open-label, randomised controlled trial. The main outcome measured was the reduction in residual depressive symptoms (Hamilton Rating Scale for Depression, HRSD-17).

\section{Results}

Mindfulness-based cognitive therapy was superior to the control condition across subgroups ( $\beta=-0.56, P<0.001)$. The interaction between treatment and subgroup was not significant $(\beta=0.45, P=0.16)$.

\section{Conclusions}

Mindfulness-based cognitive therapy reduces residual depressive symptoms irrespective of the number of previous episodes of major depression.

\section{Declaration of interest}

F.P. has received financial compensation as an independent symposium speaker from GlaxoSmithKline, wyeth, Astra Zeneca, Lundbeck, Eli Lilly, Servier and Janssen-Cilag. J.V.O. is/has been an unrestricted research grant holder with or has received financial compensation as an independent symposium speaker from Eli Lilly, BMS, Lundbeck, Organon, Janssen-Cilag, GlaxoSmithKline, Astra Zeneca, Pfizer and Servier, companies that have an interest in the treatment of mental health disorders.
Mindfulness-based cognitive therapy (MBCT) is an effective group skills-training programme originally designed to prevent depressive relapse/recurrence. Numerous studies have shown that MBCT is also associated with a reduction in depressive symptoms, ${ }^{1-8}$ which not only are harmful in the short term, but also increase risk for subsequent relapse. ${ }^{9}$ It combines methods of mindfulness training ${ }^{10}$ with features of cognitive therapy for depression. ${ }^{11,12}$ In the initial studies on MBCT, Ma \& Teasdale ${ }^{13}$ and Teasdale et al $^{14}$ found that MBCT was associated with differential risk of relapse, in that only patients with three or more episodes of depression benefitted. Based on these findings, patients with fewer than three episodes of depression are now routinely excluded $a$ priori in studies examining the effectiveness of MBCT in depression. ${ }^{3,5,15-17}$ Even when studies focused on residual depressive symptoms rather than relapse prevention, samples typically were restricted to patients with three or more depressive episodes. ${ }^{2,7,8,18}$ This restriction, however, is questionable, especially since patients with residual symptoms were actually excluded in the initial studies on differential relapse prevention. ${ }^{13,14}$ Nevertheless, National Institute for Health and Clinical Excellence guidelines ${ }^{19}$ recommend MBCT only as a relapse/recurrence prevention programme for patients with three or more prior episodes of major depression. The question arises, therefore, to what degree patients with persistent and harmful residual symptoms but only one or two prior depressive episodes currently are deprived of a treatment that may alleviate chronic complaints as well as influence the course of illness. ${ }^{20}$ The aim of the current study was to examine the efficacy of MBCT on reduction of residual depressive symptoms in a currently

'See editorial, pp. 260-261, this issue. non-depressed sample with residual depressive symptoms, explicitly comparing individuals with a history of only one or two prior episodes of major depression with individuals with three or more episodes.

\section{Method}

\section{Trial design}

The current study (MindMaastricht; trial number NTR1084, Netherlands Trial Register) was an open-label, parallel randomised controlled trial (RCT). Participants were randomised to continue with their usual treatment (if any; waiting list control condition) or to receive 8 weeks of MBCT in addition to their usual treatment (if any). Randomisation was stratified according to number of depressive episodes (one or two $v$. three or more). An independent researcher not involved in the project generated the randomisation sequence in blocks of five (using the sequence generator on www.random.org), and wrote the randomisation code in sealed numbered envelopes. Power calculations showed that the present study had $80 \%$ power for detecting a difference of medium effect size $\left(f^{2}=0.15 \text {, or } d=0.5\right)^{21}$ in differential effect of MBCT depending on number of prior episodes of depression (one or two $v$. three or more). Furthermore, our sample size of 130 participants is comparable with or greater than previous samples (such as Barnhofer et $a l^{2}$ Kingston et $a l^{7} \mathrm{Ma}$ \& Teasdale, ${ }^{13}$ Teasdale et al, ${ }^{14}$ Hargus et $a l^{18}$ ).

\section{Participant characteristics}

Adults with residual depressive symptoms after at least one episode of major depressive disorder were recruited from out-patient mental health care facilities in Maastricht and through posters in public 
spaces offering mindfulness training to people with mild depressive complaints in the context of a research project. Residual symptoms were defined as a score of seven or higher on the 17-item Hamilton Rating Scale for Depression (HRSD) ${ }^{22}$ at the time of screening. Exclusion criteria were: fulfilling criteria for a current depressive episode, schizophrenia, psychotic episodes in the previous year and recent (previous 4 weeks) or upcoming changes in ongoing psychological or pharmacological treatment. Currently depressed individuals were excluded because MBCT was developed as a relapse prevention programme and, at the time of trial preparation, the view was that current depression might complicate participation in MBCT. ${ }^{12,14}$ Relevant sociodemographic and clinical characteristics are displayed in Table 1.

\section{Sampling procedures}

All study procedures were approved by the standing Medical Ethics Committee of Maastricht University Medical Centre, and all participants signed an informed consent form. An initial telephone screening of potential participants was performed to check for availability during the study period and likelihood of meeting inclusion and exclusion criteria. A second in-person screening included administration of the Structured Clinical Interview for DSM-IV Axis I (SCID-I) ${ }^{23}$ and the 17-item HRSD by trained psychologists. Eligible participants were invited for a detailed one-to-one explanation of the study procedures, and then took part in the baseline assessment (which included the HRSD and the Inventory of Depressive Symptoms (IDS); ${ }^{24}$ see Geschwind et $a l^{20}$ for more details). After completion of all baseline assessments, the researcher allocated participants to their treatment condition based on the randomisation code in the sealed envelope. Envelopes were opened in order of sequence. No masking of treatment condition took place (although the therapists were masked to the number of prior major depressive episodes). After either 8 weeks of MBCT (see Intervention) or equivalent waiting time (in the control condition), post-assessments took place. All participants received gift vouchers worth 50 euros (mainly to compensate for the effort associated with another part of the pre- and postassessments to do with experience sampling; see Geschwind et $a l^{20}$ for more information). Participants in the control condition were offered the opportunity to take part in MBCT after the post-assessment. Participants in the MBCT condition completed follow-up assessments 6 and 12 months after the end of the training. Participant flow is displayed in Fig. 1.

\section{Intervention (MBCT)}

Content of the MBCT training sessions followed the protocol of Segal et al. ${ }^{25}$ Training consisted of weekly meetings for 8 weeks each lasting $2.5 \mathrm{~h}$ and these were run for groups of 10-15 participants (thus occasionally the groups were larger than the usual 10-12 participants per group). Assessment periods for the control participants were matched to those of the MBCT participants. Sessions included guided meditation, experiential exercises and discussions. In addition to the weekly group sessions, participants received compact discs (CDs) with guided exercises and were assigned daily homework exercises (30-60 min daily). Training sessions were delivered by experienced trainers in a centre specialised in mindfulness training. All trainers were supervised by an experienced healthcare professional who had trained with Teasdale and Williams, the co-developers of MBCT. ${ }^{25}$

\section{Measures}

Hamilton Rating Scale for Depression (HRSD)

The 17-item HRSD was administered by trained research assistants. The HRSD is a semi-structured interview designed to assess depressive symptoms over the previous week. ${ }^{22}$ It is one of the most frequently used rating scales in depression research, and sensitivity, internal, interrater and retest reliability estimates for the overall HRSD are good. ${ }^{26}$ Only the overall score was used for the analyses, and interrater reliability for the total score was high (intraclass correlation coefficient $($ ICC $)=0.97$ ). To provide information on interrater reliability, the interviewers had independently rated eight videotaped HRSD interviews with participants with varying levels of residual depressive symptoms.

\section{Inventory of Depressive Symptoms (self-rating, IDS)}

The IDS $^{24}$ is a self-rated scale, which includes 30 items rated zero to three. The scale is sensitive to change and has good psychometric properties. ${ }^{24}$ Internal consistency in the current sample was 0.85 .

\section{Statistical methods}

We undertook an intention-to-treat analysis. Primary outcome measures were residual depressive symptoms expressed as total (interviewer-rated) HRSD score. Analyses were repeated using (self-report) IDS total scores to verify whether results using interviewer- and self-rated assessments corresponded with each other. Linear regression analyses were used to examine the interaction between treatment condition (control $v$. MBCT, coded as zero and one, respectively) and number of previous depressive episodes (one or two $v$. three or more, coded as zero and one, respectively) in the model of (post) residual depressive symptoms, corrected for baseline values. Stratified by number of prior

\begin{tabular}{|c|c|c|c|c|c|}
\hline Measure & $\begin{array}{l}2 \text { or fewer prior episodes } \\
\qquad(n=71)\end{array}$ & $\begin{array}{l}3 \text { or more prior episodes } \\
\qquad(n=59)\end{array}$ & $t$-test & $\chi^{2}$ & $P$ \\
\hline Age, mean (s.d.) & $42.8(1.7)$ & $45.2(1.2)$ & 1.43 & & 0.15 \\
\hline Female gender, \% & $50(70)$ & $48(81)$ & & 3.27 & 0.07 \\
\hline Full- or part-time work, \% & $53(75)$ & $32(54)$ & & 9.63 & $<0.002$ \\
\hline IIIness/unemployment benefits, \% & $13(18)$ & $15(25)$ & & 1.45 & 0.23 \\
\hline Living with partner/own family, \% & $44(62)$ & $34(58)$ & & 0.33 & 0.56 \\
\hline Comorbid anxiety disorder (present), \% & $25(35)$ & $29(49)$ & & 3.4 & 0.06 \\
\hline Comorbid anxiety disorder (past), \% & $35(50)$ & $40(68)$ & & 6.7 & 0.01 \\
\hline Current psycho-counselling/-therapy, \% & $7(10)$ & $10(17)$ & & 2.1 & 0.15 \\
\hline Current use of antidepressants, \% & $21(30)$ & $25(43)$ & & 3.65 & 0.06 \\
\hline (Occasional) use of benzodiazepines, ${ }^{a} \%$ & $4(6)$ & $6(10)$ & & 1.01 & 0.32 \\
\hline
\end{tabular}




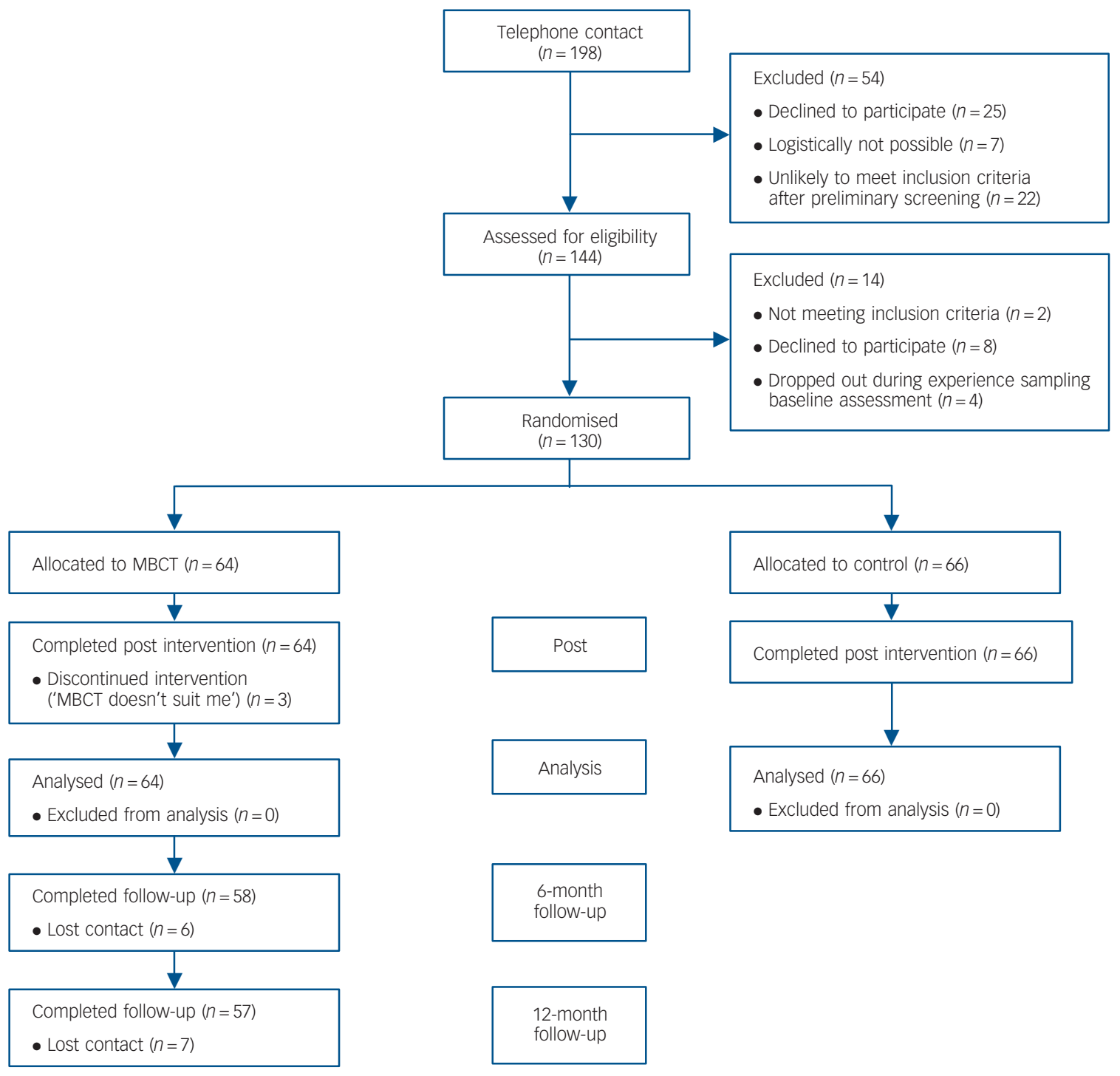

Fig. 1 Participant flow diagram.

MBCT, mindfulness-based cognitive therapy; Control, waiting list control condition. Post-assessments took place after 8-weeks of MBCT or equivalent waiting time.

depressive episodes, Cohen's $d$ and standardised effect sizes $\beta$ were calculated to express the effect of treatment on reduction of residual symptoms. Residual depressive symptoms at 6- and 12-month follow-up (MBCT group only) were compared with baseline and post-assessment scores, using paired $t$-tests. The statistical package Stata 11 for Windows was used for all analyses.

\section{Results}

\section{Participants}

Recruitment started in January 2008 and ended in February 2009; all post-intervention assessments were completed by August 2009, when the predetermined number of participants was reached. At baseline, there were no differences in sociodemographic and clinical characteristics between the MBCT and the control condition (see Geschwind et $a l^{20}$ ). Comparing participants with three or more prior major depressive episodes (hereafter: ' $3+$ ') and one or two prior episodes (hereafter: '2-') revealed that participants with $3+$ prior episodes were less likely to work and more likely to have had an anxiety disorder in the past, and were marginally more likely to have a comorbid anxiety disorder, take antidepressants, and be female (see Table 1). All analyses yielded similar results when corrected for these variables.

Table 2 shows residual depressive symptoms by subgroup (3+ v. 2-) and treatment condition. Participant flow through the study is displayed in Fig. 1. One participant completed only the HRSD but not the IDS at post-measurement. No known harm or unintended treatment effects were reported in either group. On average, participants with $2-$ prior episodes randomised to MBCT attended 7.3 sessions (s.d.=1.6), and participants with $3+$ attended 7.0 sessions (s.d. $=1.4$ ), with no significant difference in attendance between the $2-$ and $3+$ groups. Three MBCT participants attended fewer than four sessions (two in the 2and one in the $3+$ subgroups). There was no difference in daily mindfulness practice between the $2-$ and $3+$ MBCT subgroups $(t(61)=0.94, P=0.35)$ : participants in the $2-$ subgroup reported practice for $25 \mathrm{~min}$ (s.d. $=11$ ) per day on average, and participants in the $3+$ subgroup reported a daily average of $28 \mathrm{~min}($ s.d. $=10$ ). Mindfulness practice was based on self-report and calculated as minutes spent on long exercises (limited to a maximum of $45 \mathrm{~min}$, to prevent days of excessive practice skewing the average 
score), plus minutes spent on short exercises. Overall, daily mindfulness practice was positively and significantly associated with improvements in residual depressive symptoms, $t(61)=2.09, P=0.04$.

\section{Effects of treatment condition on residual depressive symptoms}

Table 2 shows pre- and post-intervention means of residual depressive symptoms by treatment condition and number of episodes. The interaction between treatment condition (MBCT $v$. control) and number of episodes $(2-v .3+)$ on reduction of residual depressive symptoms was neither large nor significant (HRSD: $\beta=0.45,95 \% \mathrm{CI}-0.18$ to $1.07, t(125)=1.41, P=0.16$ ); IDS-SR: $\beta=0.33,95 \%$ CI -0.22 to $0.89, t(124)=3.27, P=0.24$ ), indicating that the effect of MBCT does not depend on the number of depressive episodes. This was true for both the HRSD and the IDS scores. Removing the non-significant interaction term revealed a main effect of treatment condition (HRSD: $\beta=-0.56$, $95 \%$ CI -0.87 to $-0.25, t(126)=3.56, P<0.001)$; IDS: $\beta=-0.44$, $95 \%$ CI -0.71 to $-0.18, t(125)=3.27, P<0.001)$ but not of number of episodes (HRSD: $\beta=0.20,95 \%$ CI -0.11 to 0.51 , $t(126)=1.27, P=0.21)$; IDS: $\beta=0.12,95 \% \mathrm{CI}-0.15$ to 0.39 , $t(125)=0.87, P=0.39)$. Also when number of episodes was used as a continuous variable (log-transformed to prevent problems with skewness), there was no evidence for interaction with treatment condition (HRSD: $\beta=0.09,95 \%$ CI -0.22 to 0.41 , $t(114)=0.58, P=0.57$; IDS: $\beta=0.17,95 \%$ CI -0.11 to 0.45 , $t(114)=1.17, P=0.24)$. Thus, MBCT was associated with a significantly stronger reduction of residual depressive symptoms across the whole sample. Treatment effect sizes within the two subgroups are shown in Table 3.

Additional analyses on residual symptoms at 6- and 12-month follow-up (available for MBCT group only) show that residual depressive symptoms, compared with baseline, were still significantly lower at both follow-up points (HRSD: $t(57)_{6}$ months $\left.=-3.45, P=0.001 ; t(56)_{12 \text { months }}=-5.15, P<0.001\right)$. Moreover, residual symptoms at follow-up did not differ from residual symptoms at post-treatment (HRSD: $t(57)_{6 \text { months }}=0.84$, $P=0.40 ; t(56)_{12 \text { months }}=-0.54, P=0.59$; comparable results from IDS data). Figure 2 shows changes in residual depressive symptoms by treatment condition and number of episodes.

\section{Discussion}

Across the whole sample, MBCT was associated with significant post-treatment reductions of residual depressive symptoms (approximately 30-35\% reduction) in currently non-depressed participants with residual symptoms of depression, compared with a waiting list control condition (approximately 10\% reduction). Importantly, this RCT found no evidence for a stronger effect of MBCT on residual depressive symptoms in participants with three or more prior episodes compared with participants with only one or two prior episodes. This finding has implications for clinical practice because individuals with only one or two prior episodes, given results of two earlier studies, now typically are excluded from trials investigating the effects of MBCT on residual depressive symptoms. Notably, these two earlier studies examined patients without residual symptoms, and used a different outcome measure (relapse/recurrence). ${ }^{13,14}$ Consequently, it may be argued that individuals with persistent and harmful residual depressive symptoms originating from a first or second episode should not be denied access to treatment that can improve their quality of life and possibly decrease their risk of relapse/recurrence. The finding that MBCT did not selectively reduce residual depressive symptoms in individuals with three or more prior episodes of depression cannot be attributed to too low power, given that reductions in residual symptoms in the MBCT $v$. the waiting list control group, if anything, were

\begin{tabular}{|c|c|c|c|c|c|c|}
\hline \multirow[b]{3}{*}{ Measure } & \multicolumn{6}{|c|}{ Mean (s.d.) } \\
\hline & \multicolumn{2}{|c|}{ Waiting list control condition } & \multicolumn{4}{|c|}{ Mindfulness-based cognitive therapy } \\
\hline & pre & post & pre & post & 6 months & 12 months \\
\hline \multicolumn{7}{|l|}{1 or 2 prior episodes } \\
\hline Hamilton Rating Scale for Depression & $10.5(3.7)$ & $9.8(4.1)$ & $9.6(3.2)$ & $5.9(4.2)$ & $6.5(4.8)$ & $5.3(3.6)$ \\
\hline Inventory of Depressive Symptoms & $23.8(8.8)$ & $20.3(9.9)$ & $19.3(9.4)$ & $11.4(8.9)$ & $14.4(10.8)$ & $11.7(8.1)$ \\
\hline \multicolumn{7}{|l|}{3 or more prior episodes } \\
\hline Hamilton Rating Scale for Depression & $9.9(3.4)$ & $9.5(4.0)$ & $11.1(4.1)$ & $8.6(5.1)$ & $9.1(6.8)$ & $8.9(5.0)$ \\
\hline Inventory of Depressive Symptoms & $20.5(8.2)$ & $17.9(9.1)$ & $26.0(11.1)$ & $18.6(11.5)$ & $19.2(13.5)$ & $20.0(11.9)$ \\
\hline
\end{tabular}

Means (s.d.) of residual depressive symptoms by treatment condition, measurement occasion, and number of prior episodes of major depression. Baseline Inventory of Depressive Symptoms (but not Hamilton Rating Scale for Depression) scores differed significantly between subgroup treatment conditions at baseline. Follow-up data are not available for participants in the control condition, because they were offered the opportunity to take part in MBCT after post-measurement.

\begin{tabular}{|c|c|c|c|c|}
\hline Measure & $\beta(95 \% \mathrm{Cl})$ & $t$-test & d.f. & Cohen's $d$ \\
\hline \multicolumn{5}{|l|}{1 or 2 prior episodes } \\
\hline Hamilton Rating Scale for Depression & $-0.74(-1.13$ to -0.35$)$ & $3.49 * * *$ & 68 & 0.9 \\
\hline Inventory of Depressive Symptoms & $-0.60(-0.96$ to -0.23$)$ & $3.29 * *$ & 68 & 0.5 \\
\hline \multicolumn{5}{|l|}{3 or more prior episodes } \\
\hline Hamilton Rating Scale for Depression & $-0.29(-0.80$ to 0.22$)$ & 1.13 & $56^{b}$ & 0.5 \\
\hline Inventory of Depressive Symptoms & $-0.26(-0.71$ to 0.18$)$ & 1.19 & 55 & 0.6 \\
\hline \multicolumn{5}{|c|}{ 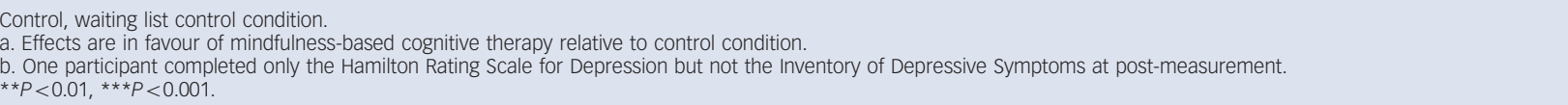 } \\
\hline
\end{tabular}




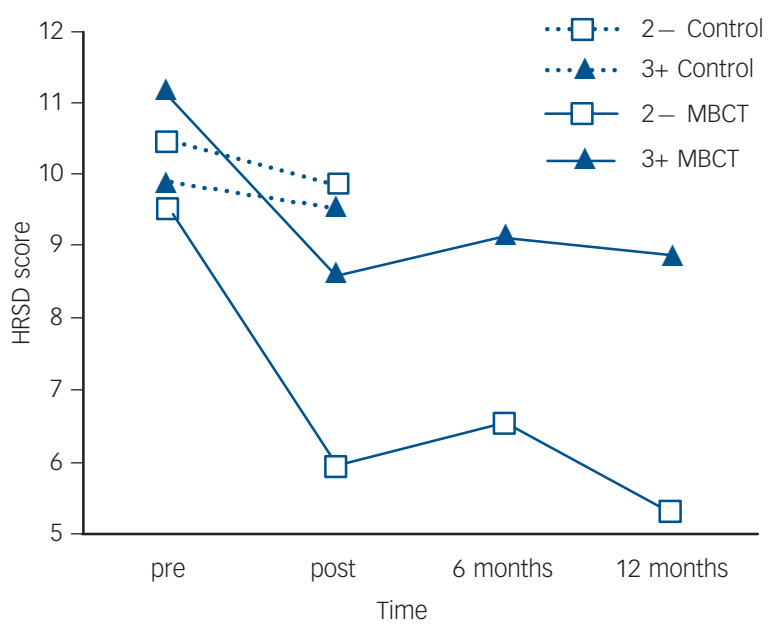

Fig. 2 Residual depressive symptoms by treatment condition and number of prior episodes.

Follow-up meetings 6 and 12 months after the end of mindfulness-based cognitive therapy (MBCT) are available only for the MBCT condition. 2-, one or two prior episodes of major depression; 3+, three or more prior episodes; Control, waiting lis control condition; HRSD, Hamilton Rating scale for Depression.

non-significantly larger in the group with one or two prior episodes than the group with three or more.

Results suggest that general vulnerability to depression may form an indication for MBCT (rather than a restriction based on the number of recurrent episodes). Vulnerability may be defined as recurrent depression (for example, Ma \& Teasdale, ${ }^{13}$ Teasdale et $\mathrm{al}^{14}$ ), or as residual symptoms, similar to the present study. Residual symptoms have a sizeable impact on quality of life and represent a major risk factor for depressive relapse or recurrence. ${ }^{27,28}$ Results of a recent trial, ${ }^{17}$ in which Segal et al investigated the role of residual symptoms during remission with regard to the efficacy of MBCT for relapse prevention, support the idea that residual symptoms may co-determine efficacy of MBCT. Mindfulness-based cognitive therapy was superior to placebo ( $74 \%$ decrease in risk for relapse) and as effective as continuation with active antidepressants only among participants who experienced residual depressive symptoms during remission ('unstable remitters'). Among participants who did not experience residual symptoms during remission ('stable remitters'), there was no difference between continuation with MBCT, active antidepressants or placebo.

\section{Clinical implications}

Clinical implications following from this trial are that MBCT treatment for residual symptoms should not be restricted to individuals with three or more prior depressive episodes, although replication is warranted. At the very least, this practice needs urgent re-examination. Reduction of residual symptoms can be expected to translate to improved quality of life as well as reduced risk for relapse, ${ }^{9,28}$ although these variables were not measured in the current study.

\section{Strengths and limitations}

Strengths of the current study include the systematic evaluation of MBCT for subgroups of patients with one or two $v$. three or more prior depressive episodes, as well as the large sample size of 71 participants with only one or two prior episodes of major depression (compared with 32 and 18 in the original relapse prevention studies ${ }^{13,14}$ ). Also, inclusion criteria were intentionally kept broad to enhance generalisability with respect to the general population of patients with depression. Finally, all randomised participants were assessed on the main outcome measure at baseline and post-intervention (without attrition), and adherence to treatment was high, with only 3 of 64 MBCT participants attending fewer than four out of eight sessions.

Some limitations also apply. First, comparison between the MBCT and waiting list control condition was only possible immediately post-treatment, because waiting list control participants were given the opportunity to attend MBCT after the post-measurements. However, 6- and 12-month follow-up data for the MBCT group suggest that improvements in residual depressive symptoms remain stable. Second, the broad inclusion criteria led to a large amount of heterogeneity in current and previous treatments, complicating fine-grained comparison of treatment between groups. On the other hand, this was an RCT, and participants were randomised within strata of one or two $v$. three or more prior depressive episodes (thus in theory ensuring an equal - or nearly equal - distribution of measured and unmeasured confounders across subgroups). Although there were no differences in sociodemographic and clinical variables between the MBCT and waiting list control conditions, participants with three or more prior depressive episodes seemed more pervasively affected by the depressive disorder, compared with participants with only one or two prior episodes (less likely to work, higher comorbidity). Nevertheless, experiencing more pervasive consequences as the number of depressive episodes increases is entirely in line with clinical reality, and attempts to cancel out such real-life differences statistically are not recommended. ${ }^{29}$ Third, the open-label, non-masked nature of the current trial may have led to biased findings. However, analyses based on interview $v$. self-report measures of residual depressive symptoms yielded similar results, and therapists were masked to participants' history of prior major depressive episodes. Fourth, limitations include the absence of MBCT competence and adherence measures, as well as the limited range of outcome measures. Moreover, this RCT took place in a specialist setting and the sample was partially recruited by posters in public spaces, raising the possibility of self-selection biases. Replication in a more traditional clinical setting, using additional outcome measures, such as relapse/recurrence and quality of life, would be desirable. Future studies may also include an active control group as well as measures of self-help (using books or the internet).

In summary, the main benefit of the current study is that it suggests that MBCT is also efficacious in individuals with only one or two prior episodes of major depression given current residual depressive symptoms. We hope that these findings, at the very least, will lead to a renewed interest as to whether or not MBCT is also indicated for individuals with fewer than three previous episodes of major depression.

\section{Funding}

Marieke Wichers was supported by the Dutch Organisation for Scientific Research (NWO) (VENI grant number: 916.76.147). Data collection was financially supported by Servier (unrestricted grant).

\section{Acknowledgements}

We thank our study participants and also Rufa Diederen, Karlijn Gielen, Marga Schnitzeler, Greet Kellens and the RIAGG Maastricht for their help during data collection, and Truda Greet Kellens and the RIAGG Maastricht for their help during data collection, and Truda
Driesen, Philippe Delespaul, Frieda van Goethem and the 4D database team for their help with data entry. 
Nicole Geschwind, PhD, Department of Psychiatry and Psychology, Maastricht University Medical Centre, The Netherlands, and Research Group on Health Psychology, CLEP, Department of Psychology, University of Leuven, Belgium; Frenk Peeters, MD, PhD, European Graduate School for Neuroscience, SEARCH Department of Psychiatry and Psychology, Maastricht University Medical Centre, Maastricht, The Netherlands; Marcus Huibers, PhD, Department of Clinical Psychological Science, Maastricht University, and Department of Clinical Psychology, VU University Amsterdam, The Netherlands; Jim van Os, MD, PhD, European Graduate School for Neuroscience, SEARCH, Department of Psychiatry and Psychology, Maastricht University Medical Centre, Maastricht, The Netherlands, and King's College London, King's Health Partners, Department of Psychosis Studies, Institute of PSychiatry, London, UK, Marieke Wichers, PhD, European Graduat School for Neuroscience, SEARCH, Department of Psychiatry and Psychology, Maastricht University Medical Centre, Maastricht, The Netherlands

Correspondence: Nicole Geschwind, Center for Learning and Experimental Psychology, University of Leuven, Tiensestraat 102, Box 3712, 3000 Leuven, Belgium. Email: nicole.geschwind@ppw.kuleuven.be

First received 28 Oct 2011, final revision 15 Feb 2012, accepted 29 May 2012

\section{References}

1 Mathew KL, Whitford HS, Kenny MA, Denson LA. The long-term effects of mindfulness-based cognitive therapy as a relapse prevention treatment for major depressive disorder. Behav Cognit Psychother 2010; 38: 561-76.

2 Barnhofer T, Crane C, Hargus E, Amarasinghe M, Winder R, Williams JMG. Mindfulness-based cognitive therapy as a treatment for chronic depression: a preliminary study. Behav Res Ther 2009; 47: 366-73.

3 Bondolfi G, Jermann F, Van der Linden M, Gex-Fabry M, Bizzini L, Rouget BW et al. Depression relapse prophylaxis with mindfulness-based cognitive therapy: replication and extension in the Swiss health care system. J Affect Disord 2010; 122: 224-31.

4 Williams JMG, Russell I, Russell D. Mindfulness-based cognitive therapy: further issues in current evidence and future research. J Consult Clin Psychol 2008; 76: 524-9.

5 Kuyken W, Byford S, Taylor RS, Watkins E, Holden E, White K, et al. Mindfulness-based cognitive therapy to prevent relapse in recurrent depression. J Consult Clin Psychol 2008; 76: 966-78.

6 Kenny MA, Williams JMG. Treatment-resistant depressed patients show a good response to mindfulness-based cognitive therapy. Behav Res Ther 2007; 45: 617-25

7 Kingston T, Dooley B, Bates A, Lawlor E, Malone K. Mindfulness-based cognitive therapy for residual depressive symptoms. Psychol Psychother 2007; 80: 193-203

8 Van Aalderen JA, Donders ART, Giommi F, Spinhoven P, Barendregt HP, Speckens AEM. The efficacy of mindfulness-based cognitive therapy in recurrent depressed patients with and without a current depressive episode: a randomized controlled trial. Psychol Med 2012; 42: 989-1001.

9 Judd LL, Paulus MP, Zeller P. The role of residual subthreshold depressive symptoms in early episode relapse in unipolar major depressive disorder. Arch Gen Psychiatry 1999; 56: 764-5.

10 Kabat-Zinn J. Full Catastrophe Living: The Program of the Stress Reduction Clinic at the University of Massachusetts Medical Center. Dell Publishing, 1990.

11 Beck AT, Rush AJ, Shaw BF, Emergy G. Cognitive Therapy of Depression. Guilford Press, 1979.
12 Teasdale JD, Segal ZV, Williams JMG. How does cognitive therapy prevent depressive relapse and why should attentional control (mindfulness) training help. Behav Res Ther 1995; 33: 25-39.

13 Ma SH, Teasdale JD. Mindfulness-based cognitive therapy for depression: replication and exploration of differential relapse prevention effects. J Consult Clin Psychol 2004; 72: 31-40.

14 Teasdale JD, Segal ZV, Williams JMG, Ridgeway VA, Soulsby JM, Lau MA. Prevention of relapse/recurrence in major depression by mindfulness-based cognitive therapy. J Consult Clin Psychol 2000; 68: 615-23.

15 Godfrin KA, van Heeringen C. The effects of mindfulness-based cognitive therapy on recurrence of depressive episodes, mental health and quality of life: a randomized controlled study. Behav Res Ther 2010; 48: 738-46.

16 Kuyken W, Watkins E, Holden E, White K, Taylor RS, Byford S, et al. How does mindfulness-based cognitive therapy work? Behav Res Ther 2010; 48 $1105-12$.

17 Segal ZV, Bieling P, Young T, MacQueen G, Cooke R, Martin L, et al Antidepressant monotherapy vs sequential pharmacotherapy and mindfulness-based cognitive therapy, or placebo, for relapse prophylaxis in recurrent depression. Arch Gen Psychiatry 2010; 67: 1256-64.

18 Hargus E, Crane C, Barnhofer T, Williams JMG. Effects of mindfulness on meta-awareness and specificity of describing prodromal symptoms in suicidal depression. Emotion 2010; 10: 34-42.

19 National Institute for Health and Clinical Excellence. Depression: The Treatment and Management of Depression in Adults (Update). Clinical Guideline 90. NICE, 2009

20 Geschwind N, Peeters F, Drukker M, van Os J, Wichers M. Mindfulness training increases momentary positive emotions and reward experience in adults vulnerable to depression: a randomized controlled trial. J Consult Clin Psychol 2011; 79: 618-28.

21 Cohen J. Statistical Power Analysis for the Behavioral Sciences. Lawrence Earlbaum Associates, 1988.

22 Hamilton M. A rating scale for depression. J Neurol Neurosurg Psychiatry 1960; 23: 56-62.

23 First MB, Spitzer RL, Gibbon M, Williams JBW. Structured Clinical Interview for DSM-IV-TR Axis 1 Disorders, Research Version. Biometrics Research, New York State Psychiatric Institute, 2002.

24 Rush AJ, Gullion CM, Basco MR, Jarrett RB, Trivedi MH. The Inventory of Depressive Symptomatology (IDS): psychometric properties. Psychol Med 1996; 26: 477-86.

25 Segal ZV, Williams JMG, Teasdale JD. Mindfulness-Based Cognitive Therapy for Depression: A New Approach to Preventing Relapse. Guilford Press, 2002.

26 Bagby RM, Ryder AG, Schuller DR, Marshall MB. The Hamilton Depression Rating Scale: has the gold standard become a lead weight? Am J Psychiatr 2004; 161: 2163-77.

27 Judd LL, Akiskal HS, Maser JD, Zeller PJ, Endicott J, Coryell W, et al. Major depressive disorder: A prospective study of residual subthreshold depressive symptoms as predictor of rapid relapse. J Affect Disord 1998; 50: 97-108.

28 Nierenberg AA, Husain MM, Trivedi MH, Fava M, Warden D, Wisniewski SR, et al. Residual symptoms after remission of major depressive disorder with citalopram and risk of relapse: a STAR*D report. Psychol Med 2010; 40 41-50.

29 Miller GA, Chapman JP. Misunderstanding analysis of covariance. J Abnorm Psychol 2001; 110: 40-8 